\title{
ON A FIXED POINT THEOREM FOR NONLINEAR $P$-COMPACT OPERATORS IN BANACH SPACE ${ }^{1}$
}

\author{
BY W. V. PETRYSHYN \\ Communicated by F. Browder, October 26, 1965
}

1. Introduction. In [5] Kaniel proved a fixed point theorem for a nonlinear quasi-compact operator in a Banach space. The purpose of this paper is to generalize and simplify Kaniel's main result and its proof to a more general class of nonlinear operators which we call projectionally-compact ( $P$-compact) and which, among others, contains completely continuous, quasi-compact, and monotone operators. From our general fixed point theorem for $P$-compact operators we then deduce in a simple way the fixed point theorems of Schauder [11], Rothe [10], Krasnoselsky [6], Altman [1], Kaniel [5], and others. In case the underlying space is a Hilbert space, we deduce (see also Kaniel [5]) some theorems on strongly monotone operators obtained by Minty [7] and Browder [2], [3], [4]. Let us add that our conditions have a form which not only admits a simpler investigation but at the same time seem to be more natural and suitable for applications to numerical functional analysis. Furthermore, the method of our proof is basically constructive. In fact, we show in [9] that it is essentially the projection method of which the Galerkin method is one of its simplest realizations. The latter methods are known [8] to play an important role in the approximate solution of operator equations.

2. Preliminary results. Let $X$ be a finite dimensional Banach space; let $B_{r}$ denote the closed ball in $X$ of radius $r>0$ about the origin and let $S_{r}$ denote the boundary of $B_{r}$. For later use we state first an essentially known fixed point theorem whose brief proof, which is based on the Brouwer fixed point theorem and the retraction mapping principle is given in [9].

TheOReM 1. Let $A$ be a continuous mapping of $B_{r}$ into $X$ and let $\mu$ be any constant. Then there exists at least one element $u$ in $B_{r}-S_{r}$ such that

$$
A u-\mu u=0
$$

provided that the mapping $A$ satisfies the condition:

$\left(\pi_{\mu}\right)$ : If for some $x$ in $S_{r}$ the equation $A x=\alpha x$ holds then $\alpha<\mu$.

1 The expanded version of this paper with detailed proofs will appear in [9]. 
Let us note that our Theorem 1 is equivalent to Theorem 1 proved in [5] by a more complicated argument. For other special cases of Theorem 1 see [9].

3. Main results for infinite dimensional spaces. In order to generalize Theorem 1 to operators defined on infinite dimensional real Banach and Hilbert spaces we assume that the Banach space $X$ has the property that there exists a sequence $\left\{X_{n}\right\}$ of finite dimensional subspaces $X_{n}$ of $X$, a sequence of linear projections $\left\{P_{n}\right\}$ on $X$, and a constant $K>0$ such that

$$
\begin{aligned}
& P_{n} X=X_{n}, \quad X_{n} \subset X_{n+1}, \quad n=1,2,3, \cdots \quad \bar{\bigcup} \bar{\bigcup} X_{n}=X, \\
& \left\|P_{n}\right\| \leqq K, \quad n=1,2,3, \cdots .
\end{aligned}
$$

REMARK 1. In this paper we shall use the symbols " $\rightarrow$ " and " $\rightarrow$ " to denote the strong and weak convergence in $X$, respectively.

For operators $A$ defined on $X$ or on a subset of $X$, we consider here only those operators $A$ which are bounded, ${ }^{2}$ i.e., operators which map bounded sets in $X$ into bounded sets in $X$.

Definition 1. A nonlinear operator $A$ will be called $P$-compact if $P_{n} A$ is continuous in $X_{n}$ for all sufficiently large $n$ and if for any constant $p>0$ and any bounded sequence $\left\{x_{n}\right\}$ with $x_{n} \in X_{n}$ the strong convergence of the sequence $\left\{g_{n}\right\} \equiv\left\{P_{n} A x_{n}-p x_{n}\right\}$ implies the existence of a strongly convergent subsequence $\left\{x_{n_{i}}\right\}$ and an element $x$ in $X$ such that $x_{n_{i}} \rightarrow x$ and $P_{n_{i}} A x_{n_{i}} \rightarrow A x$, as $n_{i} \rightarrow \infty$.

For this class of operators the following fixed point theorem is valid.

Theorem 2. Suppose that $A$ is $P$-compact. Suppose further that for given $r>0$ and $\mu>0$ the operator $A$ satisfies the condition:

$\left(\pi_{\mu}\right)$ : If some some $x$ in $S_{r}$ the equation $A x=\alpha x$ holds then $\alpha<\mu$; then there exists at least one element $u$ in $\left(B_{r}-S_{r}\right)$ such that

$$
A u-\mu u=0 \text {. }
$$

Proof. The proof of Theorem 2 depends on Theorem 1 and the following lemma.

LEMmA 1. If $A$ satisfies the conditions of Theorem 2, then there exists an integer $n_{0}>0$ such that if $n \geqq n_{0}$ and $P_{n} A x=\beta x$ for some $x$ in $S_{r} \cap X_{n}$ then $\beta<\mu$.

Proof of Lemma 1. Let us first note that in view of (3) and the

2 The much more general results analogous to Theorems 2 and 4 below (to be published elsewhere) were since obtained by the author without the assumption that $A$ be bounded. 
boundedness of $A$ there exists a constant $c>0$ such that $\left\|P_{n} A x\right\| \leqq c$ for all $x$ in $B_{r}$. If the assertion of Lemma 1 were not true, we could find a sequence $\left\{x_{n}\right\}$ with $x_{n} \in X_{n} \cap S_{r}$ and a sequence of numbers $\left\{\beta_{n}\right\}$ such that

$$
P_{n} A x_{n}=\beta_{n} x_{n}, \quad\left(\beta_{n} \geqq \mu\right) .
$$

Hence

$$
\beta_{n} r=\left\|\beta_{n} x_{n}\right\|=\left\|P_{n} A x_{n}\right\| \leqq c,
$$

i.e., $\beta_{n} \in[\mu, c / r]$ for each $n$. Passing to a subsequence, we may assume that $\beta_{n} \rightarrow \beta$ and $\beta \in[\mu, c / r]$. This and (5) imply that

$$
P_{n} A x_{n}-\beta x_{n}=\left(\beta_{n}-\beta\right) x_{n} \rightarrow 0, \quad(n \rightarrow \infty) .
$$

Since $A$ is $P$-compact, (6) implies the existence of a strongly convergent subsequence, which we again denote by $\left\{x_{n}\right\}$, and an element $x$ in $S_{r} \cap X$ such that

$$
x_{n} \rightarrow x \text { and } P_{n} A x_{n} \rightarrow A x .
$$

This and (6) imply that $A x-\beta x=0$ for $x \in S_{r}$ and $\beta \geqq \mu$ in contradiction to the condition $\left(\pi_{\mu}\right)$ of Theorem 2.

Proof of Theorem 2 Completed. By Lemma 1, we can use Theorem 1 for the finite dimensional spaces $X_{n}$ and the operators $P_{n} A$. Consequently, there exists an integer $N_{0}>0$ such that for each $n \geqq N_{0}$ there exists at least one element $u_{n}$ in $B_{r} \cap X_{n}$ such that

$$
P_{n} A u_{n}-\mu u_{n}=0 .
$$

Therefore, again by the $P$-compactness of $A$, there exists a subsequence again denoted by $\left\{u_{n}\right\}$ and an element $u \in B_{r} \cap X$ such that $u_{n} \rightarrow u, P_{n} A u_{n} \rightarrow A u$, and $A u-\mu u=0$. The last equation implies that $u \in\left(B_{r}-S_{r}\right)$ since the assumption that $u \in S_{r}$ would lead to the contradiction of condition $\left(\pi_{\mu}\right)$.

Remark 2. It is obvious that if in Definition 1 we require $p<0$ instead of $p>0$, then we get a theorem analogous to Theorem 2 . We need only consider $-A$ instead of $A$ and assume that for some $r>0$ and any $\mu<0$ instead of the condition $\left(\pi_{\mu}\right)$ the operator $A$ satisfies the condition $\left(\pi_{\mu}^{-}\right)$: If for some $x$ in $S_{r}$ the equation $A x=\alpha x$ holds then $\alpha>\mu$.

To see what type of operators belong to the class of $P$-compact operators let us first recall (following [3], [4], [5], [7]) that:

$A$ is demicontinuous if $x_{n} \rightarrow x$ implies $A x_{n} \rightarrow A x$;

$A$ is quasi-compact if $A$ satisfies the following conditions: (i) $A$ is bounded, (ii) $x_{n} \rightarrow x$ implies $P_{m} A x_{n} \rightarrow P_{m} A x$ for $m=1,2,3,4, \cdots$, (iii) if for some $\lambda>0$ the sequence $\left\{g_{n}\right\} \equiv\left\{A x_{n}+\lambda x_{n}\right\}$, where $x_{n}$ is a 
bounded sequence, is strongly convergent, then there exists a strongly convergent subsequence $\left\{x_{n_{i}}\right\}$ of $\left\{x_{n}\right\}$, (iv) if for some $\lambda>0$ the sequence $\left\{g_{n}\right\} \equiv\left\{P_{n} A x_{n}+\lambda x_{n}\right\}$, where $x_{n}$ is a bounded sequence with $x_{n} \in X_{n}$ is strongly convergent, then there exists a subsequence $\left\{x_{n_{i}}\right\}$ which is strongly convergent;

$A$ is strongly continuous if $x_{n} \rightarrow x$ implies $A x_{n} \rightarrow A x$;

$A$ is monotone increasing on the Hilbert space $X=H$ if $(A x-A y$, $x-y) \geqq 0$ for all $x$ and $y$ in $H$;

$A$ is monotone decreasing if $-A$ is monotone increasing;

$A$ is hemicontinuous if it is continuous from each line segment in $H$ to the weak topology in $H$. The following theorem whose simple proof is given in [9] specifies the relation between the operators defined above and the class of $P$-compact operators.

Theorem 3. The class of $P$-compact operators with $p<0$ includes:

(a) Completely continuous and strongly continuous operators on $X$.

(b) Quasi-compact operators in X.

(c) Hemicontinuous (and hence demicontinuous, continuous and weakly continuous) monotone increasing operators in $X=H$.

Theorem 4. Let $A$ be P-compact and suppose that there exists a sequence of spheres $S_{r_{n}}$ and a sequence of positive numbers $k_{n} \rightarrow \infty$ such that for any $\eta \geqq \mu>0$ and any $x \in S_{r_{n}}$

$$
\|A x-\eta x\| \geqq k_{n} \text {. }
$$

Then for every $f \in X$ there exists an element $x$ which satisfies the equation

$$
A x-\mu x=f .
$$

4. Special cases. In this section we show that for the Banach space $X$ satisfying (2) and (3) many of the known fixed point theorems are special cases of Theorem 2. Thus, we supply elementary and essentially constructive proofs of these theorems.

Theorem S (Schauder). If $A$ is a completely continuous mapping of $B_{r}$ into $B_{r}$, then $A$ has a fixed point in $B_{r}$.

Proof. We may assume, without loss of generality, that $A$ has no fixed points on $S_{r}$. First, by Theorem 3(a), $A$ is $P$-compact and second if $A x=\alpha x$ for some $x$ in $S_{r}$, then the condition $A\left(B_{r}\right) \subset B_{r}$ implies that $\alpha<1$. Hence by Theorem 2, $A$ has a fixed point in $\left(B_{r}-S_{r}\right)$.

In a similar way we also deduce ${ }^{1}$ from Theorem 2 the following:

Theorem $\mathrm{R}$ (Rothe). If $A$ is a completely continuous mapping of $B_{r}$ into $X$ such that $A\left(S_{r}\right) \subset B_{r}$, then $A$ has a fixed point in $B_{r}$. 
Theorem A (Altman). If $A$ is a completely continuous mapping of $B_{r}$ into $X$ such that $\|A x-x\|^{2} \geqq\|A x\|^{2}-\|x\|^{2}$ for all $x$ in $S_{r}$, then $A$ has a fixed point in $B_{r}$.

If $X$ is a Hilbert space, Theorem A was first proved by Krasnoselsky [6].

TheOREM K (KANIEL). If $A$ is a quasi-compact mapping of $B_{r}$ into $X$ such that $A x+\lambda x \neq 0$ for all $x$ in $S_{r}$ and any $\lambda>\mu>0$, then there exists an element $u$ in $B_{r}$ such that $A u+\mu u=0$.

The following apparently new comparison theorem is also valid.

Theorem 5. If $A$ and $B$ are two P-compact mappings of $B_{r}$ into $H$ such that

$$
(A x, x) \leqq\|x\|^{2} \text { and }\|A x-B x\| \leqq\|x-A x\| \text { for all } x \text { in } S_{r},
$$

then $B$ has a fixed point in $B_{r}$.

If $X$ is a Hilbert space $H$ and $A$ is a monotone decreasing operator on $H$, then one of the first important theorems of Minty [7] says essentially that if $A$ is continuous and $\mu>0$ then the operator $(A-\mu I)$ is onto. In [3], [4] Browder has proved this theorem (as well as other more general theorems) for demicontinuous and hemicontinuous operators while Shinbrot [12] established its validity for weakly continuous operators $A$. In view of our Theorem 3, Remark 2, and the fact noted in [5] that monotone increasing operators $C=-A$ satisfy the condition (9), it follows ${ }^{1}$ that these particular results of all of the above authors are immediately deducible from our Theorem 4.

\section{REFERENCES}

1. M. Altman, A fixed point theorem in Banach space, Bull. Polish Acad. Sci. 5 (1957), 19-22.

2. F. E. Browder, The solvability of nonlinear functional equations, Duke Math J. 30 (1963), 554-566.

3. - Variational boundary value problems for quasi-elliptic equations of arbitrary order, Proc. Nat. Acad. Sci. U.S.A. 50 (1963), 31-37.

4. - Nonlinear elliptic boundary-value problems, Bull. Amer. Math. Soc. 69 (1963), 862-874.

5. S. Kaniel, Quasi-compact nonlinear operators in Banach space and applications, Illinois J. Math. (to appear).

6. M. A. Krasnoselsky, Topological methods in the theory of nonlinear integral equations, State Publ. House, Moscow, 1956.

7. C. J. Minty, Monotone (nonlinear) operators in Hilbert space, Duke Math. J. 29 (1962), 341-346.

8. W. V. Petryshyn, On the extension and solution of nonlinear operator equations, (to appear). 
9. - On nonlinear P-compact operators in Banach space with applications to constructive fixed point theorems, J. Math. Anal Appl. (to appear).

10. E. Rothe, Zur Theorie der topologischen Ortnung und der Vektorfelder in Banachschen Raumen, Compositio Math. 5 (1937), 177-197.

11. J. Schauder, Der Fixpunksatz in Functionalraumen, Studia Math. 2 (1930), 171-180.

12. M. Shinbrot, $A$ fixed point theorem and some applications, Arch. Rational. Mech. Anal. 17 (1964), 255-271.

University of Chicago

\section{A PERTURBATION LEMMA}

\section{BY KARL GUSTAFSON ${ }^{1}$}

Communicated by F. Browder, September 29, 1965

1. Introduction. We will prove the following lemma and investigate some of its implications: namely, a short proof by Goldberg [1] of the basic perturbation theorem of Kato [2], avoiding previous homotopy arguments; an extension of results of Trotter and Nelson [3] for semigroup generators; and a criterion for well-posed perturbed problems in spaces that are not necessarily complete. For further references and more information, see [1], [2], and [3].

Throughout this paper all operators are linear with domains subspaces of a normed linear space $X$ and ranges subspaces of a normed linear space $Y$. If an operator $B$ perturbs an operator $T$, we assume that $D(B) \supset D(T)$.

In this section, the spaces need not be complete.

Lemma 1. Let $T^{-1}$ and $B$ be bounded operators with $\|B\|<\left\|T^{-1}\right\|^{-1}$. Then

$$
\operatorname{dim} Y / \mathrm{Cl}(R(T))=\operatorname{dim} Y / \mathrm{Cl}(R(T+B)) .
$$

Proof. ${ }^{2}$ We use the known result (e.g., see [1] for a proof) that if $\|B\|<\left\|T^{-1}\right\|-1$, then

$$
\operatorname{dim} Y / \mathrm{Cl}(R(T+B)) \leqq \operatorname{dim} Y / \mathrm{Cl}(R(T)) .
$$

1 Partially supported by a NATO postdoctoral fellowship.

2 Concerning this little result, let $\|B\|<\alpha\left\|T^{-1}\right\|^{-1}$. The author appreciates discussions with Dr. Seymour Goldberg, who proved it for $\alpha=1 / 2$ in his lectures. The main trick in the proof can be seen for the case $\alpha=3 / 4$. The author also appreciates the aid of $\mathrm{Mr} \mathrm{J}$. Kuttler in extending the result from $\alpha=3 / 4$ to $\alpha=7 / 8$. 